\title{
MACKEY COMPACTNESS IN BANACH SPACES
}

\author{
JOE HOWARD
}

\begin{abstract}
If $A^{\prime}$, a subset of a conjugate Banach space $X^{\prime}$, is sequentially compact in the Mackey topology $\left(\tau\left(X^{\prime}, X\right)\right)$, then $A^{\prime}$ is conditionally compact in the Mackey topology. The converse is not true.
\end{abstract}

A subset $A$ is conditionally compact if its closure is compact; $A$ is sequentially compact if each sequence of elements of $A$ contains a subsequence converging to an element of the Banach space $X$. It is well known (Eberlein-Smulian Theorem) that sequential and conditional compactness are equivalent in the weak topology $\left(\sigma\left(X, X^{\prime}\right)\right)$ of a Banach space $X$; and that sequential compactness implies conditional compactness (but not conversely) for the weak-star topology $\left(\sigma\left(X^{\prime}, X\right)\right)$. It is natural to ask what is the relation, if any, between conditionally compact and sequentially compact sets in the Mackey topology $\left(\tau\left(X^{\prime}, X\right)\right)$ of $X^{\prime}$ ? Recall that the Mackey topology on $X^{\prime}$ is that topology generated by the polars of all convex, balanced, and weakly compact sets of $X$ (see [4]).

LEMMA 1 (GROTHENDIECK [2, p. 134]). Let $X$ be a Banach space and $A^{\prime}$ be a subset of $X^{\prime}$. The following conditions are equivalent:

(a) $A^{\prime}$ is Mackey conditionally compact.

(b) $\lim _{n} \sup _{A^{\prime}}\left|x^{\prime}\left(x_{n}\right)\right|=0$ for every sequence $\left\{x_{n}\right\}$ in $X$ which weakly converges to 0 .

THEOREM 2. Let $A^{\prime} \subseteq X^{\prime}$ be Mackey sequentially compact. Then $A^{\prime}$ is Mackey conditionally compact.

Proof. Let $\left\{x_{n}\right\} \subseteq X$ weakly converge to 0 and $\left\{x_{n}^{\prime}\right\}$ be a sequence in $A^{\prime}$. We show $\lim _{n} x_{n}^{\prime}\left(x_{n}\right)=0$. Let $W$ be the closed, balanced convex hull of $S=\left\{x_{n}, 0\right\}$. Now $W$ is the closed convex hull of the set $W_{0}=\{\alpha s: \alpha$ a scalar, $|\alpha| \leqq 1, s \in S\}$, and $W_{0}$ is the image under the multiplication map of the

Received by the editors November 15, 1971 and, in revised form, May 11, 1972.

AMS (MOS) subject classifications (1969). Primary 4610, 4601.

Key words and phrases. Mackey topology, conditionally compact, sequentially compact.

(c) American Mathematical Society 1973 
set $\{|\alpha| \leqq 1\} \times S$, which is itself compact in the product space (scalars) $\times$ ( $X$, weak topology). Thus, $W_{0}$ is weakly compact, so $W$ is weakly compact by the Krein-Smulian Theorem [1, p. 434]. Since $A^{\prime}$ is Mackey sequentially compact, there exists a subsequence $\left\{x_{k}^{\prime}\right\}$ of $\left\{x_{n}^{\prime}\right\}$ which converges uniformly on $W$. Actually, we can say every subsequence of $\left\{x_{n}^{\prime}\left(x_{n}\right)\right\}$ has a subsequence converging to 0 . Therefore, $\left\{x_{n}^{\prime}\left(x_{n}\right)\right\}$ converges to 0 and, by Lemma $1, A^{\prime}$ is Mackey conditionally compact.

The following is an example of a weak-star compact set which is not weak-star sequentially compact.

EXAmple 3. The unit sphere $S^{\prime \prime}$ of $l_{\infty}[0,2 \pi]$ is not weak-star sequentially compact.

PROoF. Define a sequence in $S^{\prime \prime}$ by $x_{n}^{\prime \prime}(\gamma)=\sin n \gamma$ for all $\gamma$ in $[0,2 \pi]$ and for $n=1,2,3, \cdots$. Suppose $\left\{x_{n}^{\prime \prime}\right\}$ has a weak-star Cauchy subsequence $\left\{x_{n_{k}}^{\prime \prime}\right\}$. Then at least $\lim _{n_{k}} \sin n_{k} \gamma$ exists for each $\gamma$ in $[0,2 \pi]$. But this is not possible [3, p. 143].

EXAMPLE 4. If $A^{\prime} \subseteq X^{\prime}$ is Mackey conditionally compact, then $A^{\prime}$ is not necessarily Mackey sequentially compact.

Proof. Consider the unit sphere $S^{\prime \prime}$ of $l_{\infty}[0,2 \pi]$. By Example 3, $S^{\prime \prime}$ is not weak-star sequentially compact; and hence, cannot be Mackey sequentially compact since the Mackey topology is stronger than the weak-star topology [4, p. 248]. Since weak and norm convergence correspond in $l_{1}[0,2 \pi], S^{\prime \prime}$ is Mackey conditionally compact by Lemma 1 .

We show in the following that if $X$ is either reflexive or separable, and if $A^{\prime} \subseteq X^{\prime}$ is Mackey conditionally compact, then $A^{\prime}$ is Mackey sequentially compact.

Proposition 5. Suppose $X$ is reflexive and $A^{\prime} \subseteq X^{\prime}$ is Mackey conditionally compact. Then $A^{\prime}$ is conditionally compact (and hence, Mackey sequentially compact).

Proof. Since $X$ is reflexive, the $\tau\left(X^{\prime}, X\right)$-topology is the same as the $\tau\left(X^{\prime}, X^{\prime \prime}\right)$-topology on $X^{\prime}$. But this is the same as the norm topology on $X^{\prime}$ [4, p. 249].

Proposition 6. Let $X$ be separable. Then if $A^{\prime} \subseteq X^{\prime}$ is Mackey conditionally compact, $A^{\prime}$ is Mackey sequentially compact.

Proof. Consider the set $\mathrm{cl}\left(A^{\prime}\right)$ where cl denotes the closure in the Mackey topology. $\operatorname{cl}\left(A^{\prime}\right)$ is Mackey compact, the weak-star topology is coarser than the Mackey topology, and both topologies are Hausdorff; hence, they agree on $\operatorname{cl}\left(A^{\prime}\right)$. Since the weak-star topology is metrizable on bounded sets [1, Theorem V.5.1], the result follows.

I wish to thank D. G. Tacon for Example 3 and the referee for his helpful suggestions. 


\section{REFERENCES}

1. N. Dunford and J. T. Schwartz, Linear operators. I: General theory, Pure and Appl. Math., vol. 7, Interscience, New York, 1958. MR 22 \#8302.

2. A. Grothendieck, Sur les applications linéaires faiblement compactes d'espaces du type $C(K)$, Canad. J. Math. 5 (1953), 129-173. MR 15, 438.

3. W. Rudin, Principles of mathematical analysis, 2nd ed., McGraw-Hill, New York, 1964. MR 29 \#3587.

4. A. Wilansky, Functional analysis, Blaisdell, Waltham, Mass., 1964. MR 30 \#25.

Department of Mathematics, Oklahoma State University, Stillwater, ОKLAномA 74074 